Type of the Paper (Article, Review, Communication, etc.)

\title{
Attention-based YOLOv4 Algorithm in Non-destructive Radio- graphic Testing for Civic Aviation Maintenance
}

\author{
Zhi-Hao Chen ${ }^{1 *}$ and Jyh-Ching Juang ${ }^{2}$
}

\author{
National Cheng Kung University, Tainan, 701 Taiwan; n28061072@gs.ncku.edu.tw \\ Zhi-Hao Chen is Serving the Air Force Institute of Technology, Kaohsiung, 820 Taiwan \\ * Correspondence: n28061072@gs.ncku.edu.tw; Tel.: +886-6-2757575(ext.62400)
}

\begin{abstract}
The aim of this paper is to use deep learning tools to innovate pre-trained object detection models to improve the accuracy of non-destructive testing (NDT) of civil aviation maintenance. First, this thesis classifies object defects for NDT, such as cracks, undercut, etc. Nowadays, thesis surveys innovation deep-learning methods technology is used to improve the defect detection performance inferencing capability, increase the accuracy and efficiency of automatic identification which in enhanced the safety and reliability of aircraft fuselage in future, mark hidden cracks and solve the challenges that cannot be identified by manual inspection. Second, recent mainstream techniques the YOLOv4 neural network to the graphics card GPU core operator to speed up the recognition of defect images is being applied to the non-destructive inspection process of aircraft maintenance on A, C and D-Level, fully validating the deep learning model's powerful defect detection target capability. The attention-based YOLOv4 algorithm is improved by applying a onestage attention mechanism to the YOLOv4, thereby improving the accuracy of the innovation model. Finally, thesis improved YOLOv4 based on an attention mechanism is proposed for object detection NDT via the deep learning method to effectively improve and shorten the inspection anomaly detection method for automatic detection sensor systems.
\end{abstract}

Keywords: CNN, YOLOv4, NDT, Object Detection.

\section{Introduction}

This thesis purpose to mitigate the risk of aircraft accident, it is thus of paramount important to perform quality-assured civic aircraft maintenance inspection, manufacturing and overhaul. Indeed, if the inspection and maintenance tasks of aircraft are negligent, flight safety problems may result. This is evidenced in the event of a Boeing 737-700 passenger aircraft in April 2018 as Figure 1 [1] In this event, a serious accident occurred when the engine blade ruptured and pierced the cabin of the aircraft, damaging the fuselage and causing injury to the passenger on the spot. The main cause of the accident was that a proper inspection was not implemented when the aircraft and engine was repaired in the plant, and the hidden fatigue defects in the engine blades could not be detected by the inspector. Once engine blades with hidden cracks had exceeded their service life, fatigue cracks on metal surfaces cause cracks to continuously expand. It is thus desired to augment the existing inspection procedure with artificial intelligence to recognize potential cracks in an aero engine. Therefore, benefiting on the aviation factory automatic technology for the mechanism applications for NDT. Today, we reach the first wave aviation industry approach to AI in the deep learning technology where can see that AI on object detection ability mechanism solutions must be integrated into the NDT identification capabilities. Thus, the AI-based used on automation sensors and low false accuracy are key point for safety, but also essential is the efficient use of AI technological. As a result, this solution improved YOLOv4 model enables faster and more accurate understand repairs. We are certain that the feature analysis carried out is more effective in modeling the proposed method novel on improved deep learning based on the YOLOv4 algorithm of 
attentional mechanisms. The results are available in a few seconds after NDT examinations, resulting in greater saving workload during automatic improved renew model sensor system periods in comparison to the current automatic NDT inspection efforts. This thesis algorithm improves recognition speed, enables real-time detection and maintains high accuracy. However, most of the aviation maintenance manual solder processes are resorted to industrial radiography find defects in internal welds.

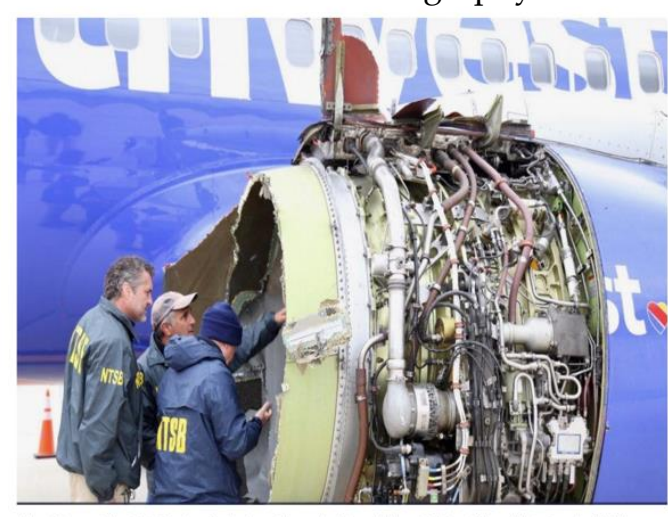

By Ashley Halsey III and Faiz Siddiqui

By Ashley Halsey
April 18, 2018
FAA, EASA Order More Frequent CFM56-7B Fan Blade Inspections

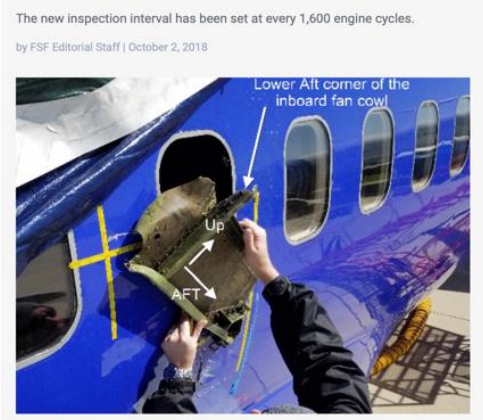

The U.S. Federal Aviation Administration (FAA) and the European Aviation Safety Agency (EASA) both have issued updated aimworthiness directives (ADS) requiling more frequent CFM International CFM
inspections. The new EASA AD is effective OCc. 5 and the FAA AD is effective Oct. 16.

Figure 1. Southwest Airlines Boeing 737-700 Engine Failure.

Using on deep learning technology improve with digitalization analytics, the value of predictive maintenance has been recognized by major players in the industry, the volume of data handled by production and maintenance companies is steadily increasing, and with it the need for artificial intelligence to process this data. Therefore, in order to reduce the risk of aircraft accidents, quality assure aircraft general inspections, manufacturing and overhauls are of paramount importance. Indeed, negligence in the inspection and maintenance of aircraft repair can lead to flight safety issues. Today, the use of artificial intelligence (AI) technologies in the aviation industry includes the development of digital twins in manufacturing, the introduction of the image sensors in the production chain, and the development of predictive maintenance, where the need for large amounts of data and the identification of low signals definitely requires the use of AI. Nowadays, global engine manufacturers are no longer really selling the engine and spares, but the number of flight hours being used. Major companies in the industry have already acknowledged the value of predictive maintenance. For example, General Electric Aircraft Engines (GEAE's Aircraft Maintenance Analysis System) is used by more than a thousand customers, and the system constantly monitors the health of the aircraft and transmits fault or warning information to ground control centers, providing quick access to maintenance documentation and troubleshooting steps ranked according to probability of success.

The experiment result creates an innovation CNN model of a fast-detecting speed of an object detector in NDT systems and optimization for GPUs parallel computations, rather than the low computation volume theoretical indicator (BFLOP) [2] suitable for aviation maintenance which operates in real-time on a conventional GPU. The YOLOv4 object detection in deterministic manner state-of-the-art method using techniques by convolution feature extraction make YOLOv4 more efficient and suitable for single GPU training, including, PAN [3], SAM [4], CBN [5], etc. This thesis modify machine learning detector is usually composed of two basic system parts, a head net which is used to predict classes and bounding boxes of objects and a backbone net which is pre-trained on ImageNet [6]. As the result, this innovation model attention-based training mode in NDT X-ray image maximizing generation to variational lower boundary. Therefore, contribute to reduce the risk of aircraft accidents, it is vital that quality assured engine inspections, manufacturing and overhauls are carried out. Indeed, negligence in the inspection and maintenance tasks of aircraft engines can lead to flight safety problems. 
In Section 2, the Materials and Methods is described, the related literature on AIbased, X-ray image datasets, improve the attentional mechanisms YOLOv4 algorithm [7] and multiple deep learning algorithms use for object identification with classification are discussed. The adopted improve R-CNN and YOLOv4 are then delineated and some modifications to existing deep learning neural network are highlighted. In Section 3, the experiment process and methods have discussed about modify the attention-based YOLOv4 algorithm structure, which hidden layers were modified one-stage model structure layers. So that, the compute faster on attentional mechanisms YOLOv4 algorithm. In Section 4, the experimental results are discussed. The experiment results indicate that the proposed AI-based inspection method can indeed effectively augment the overall inspection capability. Finally, some conclusions are given in Section 5 .

\section{Materials and Methods}

According to the FAA's Maintenance Review Board (MRB) [7], each online civic aircraft should pass the period A, B, C, and D level checks for work, including maintenance, repair, and overhaul (MRO) [8]. Civil aircraft structural and engine component parts will be inspected for hidden cracks within the internal structure of each component using nondestructive testing (NDT). Also, civic aircraft undergoing D-Level check maintenance are inspected at the factory and each structural component must pass a non-destructive test. There are two methods of non-destructive testing: digital radiographic testing (RT) [9] and computed tomography testing (CT) relies on the deep learning model to improve the existing techniques of $\mathrm{X}$-ray image inspection. Therefore, in order to reduce the risk of aircraft accidents, it is vital that quality assured civic aircraft under NDT inspections, periodic checking, parts manufacturing and overhauls are carried out. Indeed, negligence in the major parts inspection and maintenance tasks of civic aircraft can lead to major aviation safety problems. The National Transportation Safety Board's (NTSB) Global Aviation Safety Bulletin [10] states that "a single abnormal engine can cause a serious aircraft accident". An unfortunate accident, which was demonstrated by the Boeing 737-700 airliner incident that occurred in April 2018. In this incident, a serious accident occurred when an engine blade ruptured, puncturing the aircraft's cabin and damaging the fuselage, causing on-the-spot injuries to passengers. The main cause of the accident was that the aircraft engines were not properly inspected when they were serviced at the factory and the hidden fatigue of the broken engine blades could not be detected by the inspectors. Once an engine blade with hidden cracks has exceeded its service life, fatigue cracks on the metal surface can lead to ever-widening cracks. It is therefore desired to augment existing inspection procedures with artificial intelligence to identify potential cracks inside parts.

In order to ensure the NDT automatic classified high accuracy, the deep learning model is to use convolutional architecture for fast feature embedding (CAFFE) [11] driven structure on basically framework to provide training and testing of NDT with one-stage detector defect paradigms for X-ray images. In the case of high manual failure rates in NDT, the limited number and insufficient type of samples of radiographic inspection defect data can therefore introduce another serious failure in the performance accuracy of the training model to cope with multiple inspections. Preliminary experiment is used fast region based convolutional neural network (Fast R-CNN) driven model to enhance and improve existing automated non-destructive testing (NDT) diagnostics [12]. Fortunately, aircraft maintenance technical managers inspired by AI technology improve recent work operation manual, which apply on aircraft production and maintenance that automatically learns to describe the content of X-ray files images defect area mark, benefiting on the attention-mechanism on feature maps applications for NDT technology test improve automated non-destructive testing (NDT) enables faster and more accurate understand repairs.

In this section, we begin by briefly providing an overview of the related literature on automatic inspection of aerial engine using radiography testing (RT) [9] on deep learning model. Then, we introduced the improve R-CNN model (a two-stage network), attentionbased YOLOv4 model (a one-stage network), and multiple DL algorithms use for object 
identification with classification are discussed. The adopted improved R-CNN and YOLOv4 model are then delineated and some modifications to existing neural network net are highlighted.

\subsection{Related Work}

In recent years, the European Civil Aviation Organization (ICAO) has been recommending the integration of AI technology into civil aviation maintenance to improve overall safety and comfort [13]. The world's largest civil aircraft manufacturers, Airbus and Boeing, have been using AI technology to test aircraft manufacturing and maintenance work since the early 20th century. The world's largest civil aircraft manufacturers, Airbus and Boeing, as well as the largest civil airliner manufacturer, have been using AI technology to test aircraft manufacturing and maintenance work since the early 20th century. Artificial intelligence techniques have been used extensively in the aviation industry, such as online booking, customer service, management, aircraft maintenance and engine repair, gradually replacing some of the manual tasks. This paper focuses on an innovative study of the application of artificial intelligence deep learning object inspection technology combined with non-destructive radiographic inspection in maintenance work in the aviation industry.

Recent years, some papers are talking about the two-stage network compare with one-stage network which is more suitable for best object detection prediction box algorithm for digital radiography testing (RT) cracks feature map [9]. Let's take a look at the main components of a modern one-stage object detector. This thesis will focus on the former ones about the one-stage detectors. Improving clutter in the image leads to getting more accurate recognition than can be done by the more advanced standard visual inspection of recognizing patterns in the image. Furthermore, the R-CNN is primarily intended as a classifier, which does not predict object boundaries (except for refinement via bounding box regression). Although, traditional Yolo-based object detection model has onestage detectors as like R-CNN with two-stage detectors on deep network uses based on $\mathrm{R}-\mathrm{CNN}$ algorithm to training into convolutional neural network layers export into the classify proposal regions into object classes and back-grounds the improve accuracy of the YOLOv4 depends on the performance of the region proposal module, only consists of four region modules, namely the input, backbone, neck, and dense prediction [14], etc. So, some region modules composed from R-CNN model be update to YOLOv4 neural network. It is also possible the next step is to make a two-stage object detector a one-stage object detector, such as DetectNet [15], the most representative models are YOLO [16, 17] and SSD [13]. According to the literature experimental models developed in recent years for object detectors tend to insert some layers between the backbone and the head, and these layers are usually used to collect feature maps from different stages, it can be called the neck of object detector. Usually, a neck is composed of several bottom-up paths and several top-down paths. In the first step, one film of $640 \times 480$ pixels each in .txt file format was used, and then over 6,000 X-ray images were updated into the input region model. In the second step, the YOLOv4 model was improved on the basis of the R-CNN model with the addition of a backbone layer and a neck layer. Finally, the improved YOLO object detector model was named the head-layer YOLOv4 model dense prediction improved single-stage detector. Although YOLOv4 is a one-stage detector, there are also one-stage detectors, such as the R-FCN and SSD, which are accurate but slow.

The innovation YOLOv4 model is a deep full convolutional neural network of the proposed region and based on a R-CNN detector using the proposed region. General Architecture of the whole system is a unified object detection network. Recently, certain wellknown deep learning head objection detection model have demonstrated neural networks with a design model based on deep learning for object damage recognition methods. Compared to traditional machine learning methods, YOLOv4 model have a powerful ability to explore object detection and learn representations from the entire image. This modern detector for deep learning detector consists of a backbone pre-trained on the Darknet53 [18] network and a head for predicting object classes and object bounding boxes. These 
innovative deep learning models can quickly detect the location of defects in material components inside civil aircraft fuselage. An expanded convolutional neural network chain is proposed by applying plug in the spatial pyramid pooling (SPP) block over the CSPDarknet53 backbone [18], and these are used to extend the receptive field to further extract rich information about defects NDT in civic aircraft fuselage and engine parts. The convolutional neural network chain model integrated the global and local information of the feature map to locate the engine defect defection in the SPP plane more precisely and accurately. Using the YOLOv3 (anchor based) to extract the region of interest (ROI) [19] to detects composite material hidden cracks from single and multiple NDT images with high reliability in SPP additional module, and then used the global spatial information of the ROI to detect the anatomical structures. To increase the speed of detection, the innovative deep learning model first detects hidden cracks in the material where the fuselage and engine components of the aircraft are identified as broken in focus. We propose a method of improved deep learning based on R-CNN that a simple method for creating bypass nets based on the remaining layers of the encoder-decoder neural block. Based on an improved YOLOv4, this innovative deep learning model is unique and simple to code for fast detection of material defects such as cracks. This region is highlighted with a red square for crack, and manual labeled damage is marked with solid red lines, for the position, size, shape, and direction. Our automatic defect detection system can identify multiple cracks in X-ray images and mark them between civil aircraft fuselages and engine blades, as well as distinguishing between eight types of cracks. The innovation deep learning model is a YOLOv4 (one-stage detector) structure, with region-based detectors have a pretty impressive frame per seconds (FPS) [9] using lower resolution images at the cost of accuracy demonstrate a small accuracy advantage.

This paper selects which deep learning neural network structure is the most suitable experimental structure for improving the accuracy and speed of object recognition in nondestructive testing. So that, this paper present experimental results on the proposed method. The most important question is not which detector is the most suitable, which may not be answered. Therefore, the real question is which detector and what configurations give us the best balance of speed and accuracy that your application needed. In general, the two-stage network model is a more accurate architecture than the one-stage-network model, even though R-CNN and R-FCN are faster networks, but not more accurate or faster than the YOLOv4 model. Both Faster R-CNN and R-CNN model can take advantage of a better feature extractor, but also it is less significant with the SSD and DetectNet algorithm. The one-stage network model performs pretty well even with a simple extractor, that can even match other detectors' accuracies using more better extractor. For the reason, we finally choice the YOLO structure to modify and improve the innovation deep learning model more enables faster and more accurate. The thesis uses PANet as a parameter aggregation method for different backbone levels for different detector levels than the FPN used in YOLOv3. Finally, we choose the SPP add-on module, the CSPDarknet53 backbone, the PANet path aggregation neck and the YOLOv3 (anchor-based) [2] head as the architecture for YOLOv4 neural network.

\subsection{Data Preparation}

A collection of X-ray images from a civil aircraft maintenance plant in Taiwan. The plant specialises in the repair of various types of civil aircraft components and engines, such as helicopters, transport and cargo aircraft described in the paper. The airline uses radiation scanner equipment for non-destructive testing, as shown in Figure 2 for the Xray machine used at the facility. The main components of the aircraft fuselage are almost all titanium alloy and aluminum alloy, in which the essential physical properties of the materials are the characteristics of special crystal elements such as low crystal density, high stress resistance, heat resistance, and corrosion resistance. Typically, parts of an overhauled civic aircraft fuselage parts and engine are scanned by a Radiographic Test (RT) equipment and the resulting X-ray images are inspected. Level D maintenance checks in airline workshops are carried out by RT machine, which scans and inspects the resulting 
X-ray images over a period of one month. Each civil aircraft fuselage component and engine contains 300 parts and a total of 6,000 X-ray images can be collected in one month. Hence, automatic inspection by augmenting AI techniques may potentially reduce the workload of human inspectors and assure a consistent quality. The airline company factory also has NDT equipment with illustrates the X-ray machine that is used in the airline D-level check plant. Typically, parts of an overhauled engine are scanned by a RT equipment and the resulting $\mathrm{X}$-ray images are inspected.

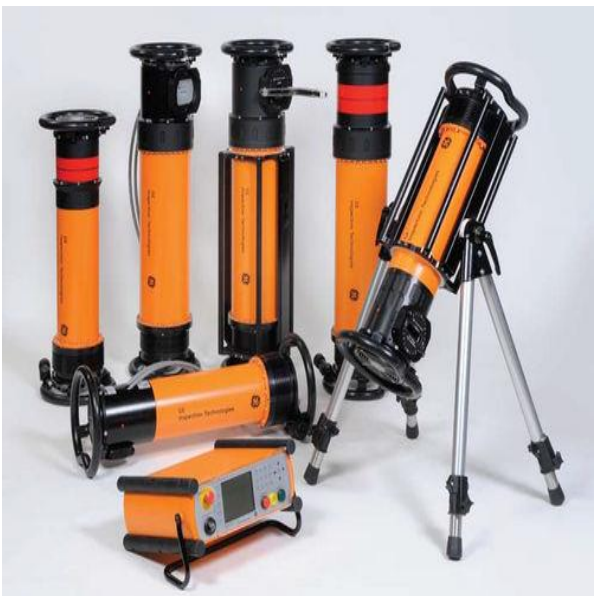

(a)

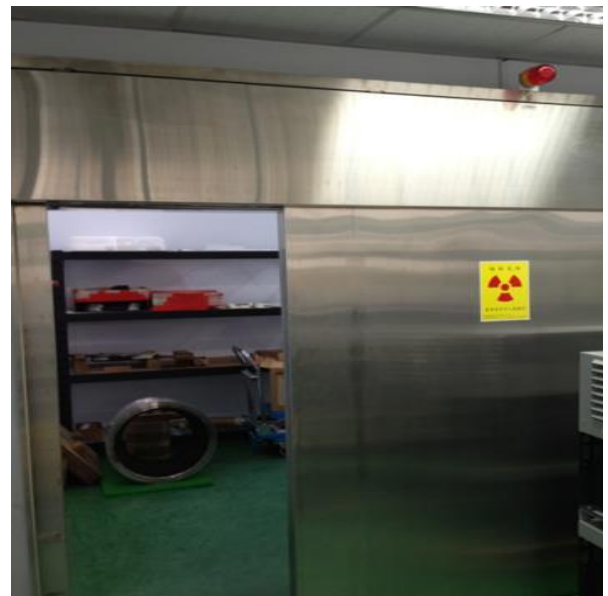

(b)

Figure 2. (a) Radiographic Test Equipment; (b) Radiographic Test Compartment.

All training and testing image tasks data sets were taken from the archives of civic aircraft fuselage and engine repair records. Next, preprocessing using the Python toolkit's image processing library, Python Imaging Library (PIL), was executed to convert the image file format and adjust the output $640 \times 480$ pixels each in size to a universal lightning memory-mapped database (LMDB) data stored in a .txt file data format [20]. Because the aircraft parts material is a superalloy composition, the crystal structure is intricate and complicated. It is easy to have shadow overlap when the sharpness of the X-ray image is poor. The aircraft company repair plant had accumulated a data gallery of civic aircraft and engine parts of X-ray images as depicted in Figure 3.

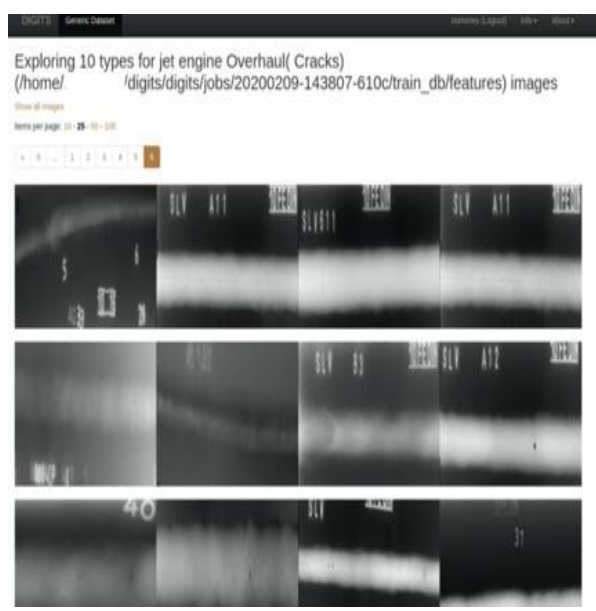

(a)

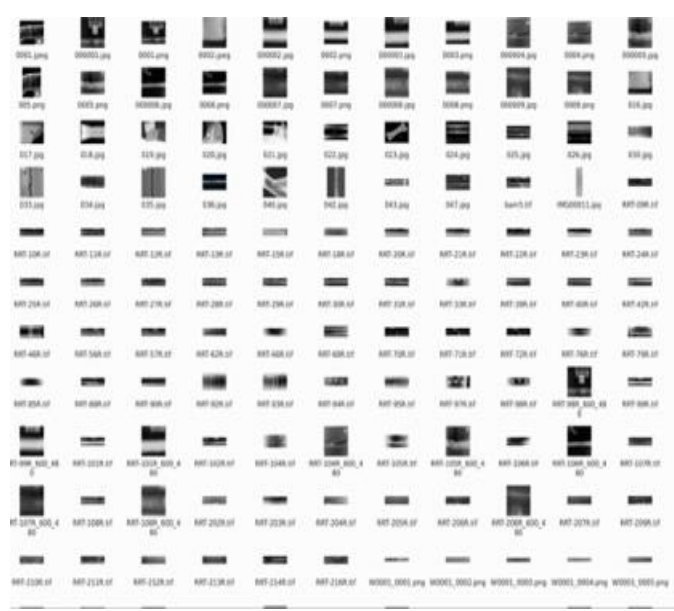

(b)

Figure 3. (a) Universal Lightning Memory-mapped Database (LMDB) format; (b) NDT X-ray Files.

The task of the technician in visual inspection is thus highly demanding, as she or he may also be subject to fatigue and psychological conditions. As a result, the aviation industry including companies such as Pratt \& Whitney, GE, and Honeywell, has attempted 
to automate the inspection process to reduce the burden of human inspector and assure the quality. An automatic inspection system can assist the inspector to identify the features and defects in X-ray images by marking the object localization window and bounding box automatically. The aircraft must not have any damage or cracks when operating at full power and high thrust. It is therefore very important that inspections are carried out with quality and efficiency in mind.

\section{Experiment Process and Methods}

In this article, Artificial Intelligence (AI) based image processing technology is combined with NDT technology to improve the inspection tasks of aircraft structural parts and engine components. In the past, some renowned manufacturer of civil aircraft and cargo planes reached the goal this year of updating its factory NDT machinery and equipment to help engineers visually inspect all X-ray images faster, with the aim of assisting NDT engineers to reduce the burden of judging images for visual inspection. As long times work fatigue can also occur mistake during manual work. Now the Automated inspection by artificial intelligence in automated assembly plants can support the engineer in his detection work, and all fuselages RT inspection are being carried out in the plant with greater speed and efficiency. Today, aviation automation shops worldwide are using artificial intelligence technology to assist engineers with difficult non-destructive testing tasks, particularly in aircraft fuselage and engine maintenance, to improve flight safety. The focus is on deep learning techniques for artificial intelligence in order to achieve better speed and accuracy in RT detection.

The contribution of this paper is the completion of a dataset of over 6,100 labelled Xray images of civil aircraft and engines using deep learning techniques for machine learning computer vision applications, and the successful compilation of a unique deep learning framework model for a one-stage detector defect paradigm. The results of the thesis helped civil companies' aircraft and engine factories to update their machine capabilities for defective object detection and segmentation of X-ray images, successfully achieving a learning accuracy of $80 \%$ for the deep learning net model we designed. The end result was just as we predicted, the AI model could be easily and cheaply estimated to be installed in connection with the X-ray image machine, successfully saving the data to SD disks. We successfully updated the plant machine and retrained a small model for deployment on the NVIDIA ${ }^{\circledR}$ Jetson ${ }^{\mathrm{TM}} \mathrm{TX} 2$ platform. The image dataset was fed into an experimental YOLOv4 model, where the deep learning model's defective object recognition module was in LMDB data stored in a .txt file data format and the YOLOv4 model was trained to object bounding boxes, recognizing a variety of sample-tagged defective feature models.

\subsection{Data Preparation}

The aircraft company repair plant has accumulated a data gallery of RT images. In the LMDB dataset, defects can be categorized as either (a) slag inclusion, (b) undercut, (c) incomplete penetration, (d) blowhole, (e) cracks, (f) incomplete fusion, (g) welding spatter, or (h) porosity as depicted in Figure 4. The X-ray images in the dataset are converted into LMDB format and stored for deep learning application. In the dataset, some $\mathrm{X}$-ray images in different light conditions and resolutions have been labeled with the above defects. An effort is made to prepare the labeled data in terms of innovation DL model [5]. In this endeavor, the 8 types of label classes are stored in the first row of the category table string over 6,100 labeled X-ray images dataset as show in Figure 5. 


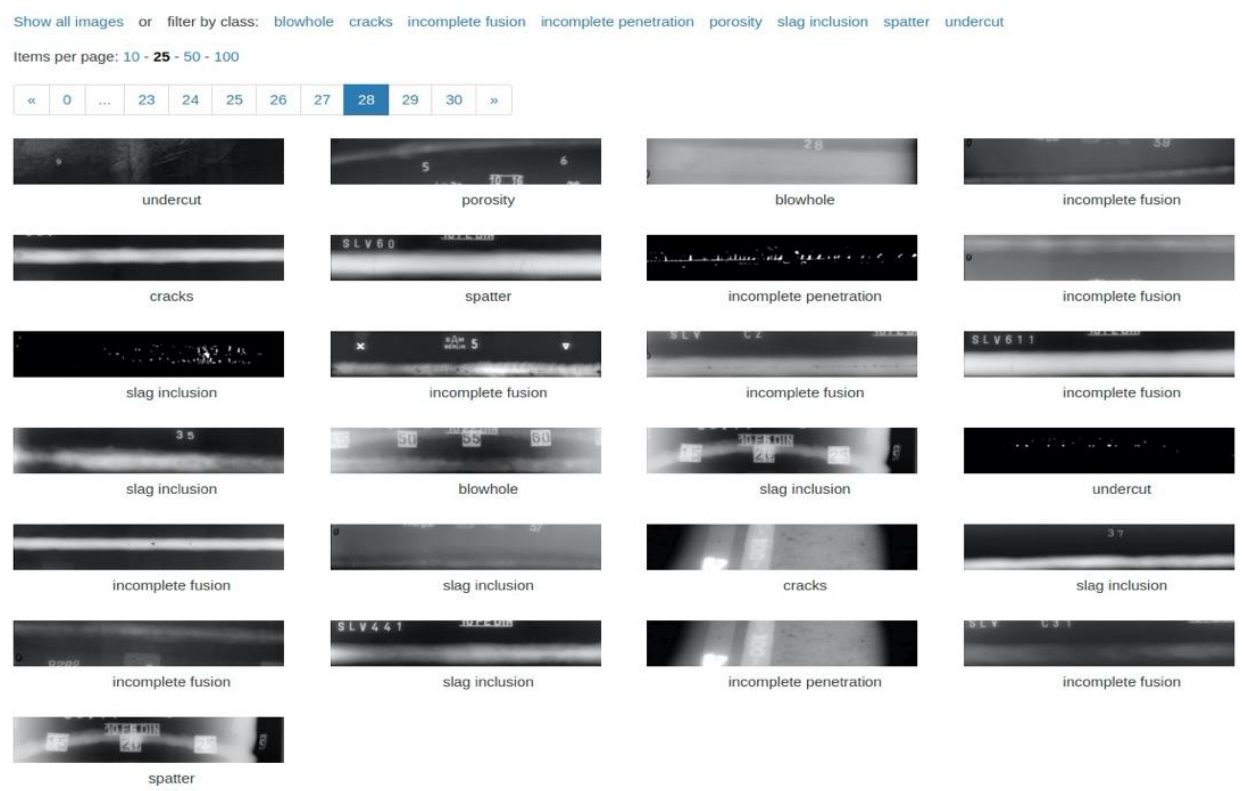

Figure 4. Differentiates Types of Defect Rating Experimental Parameters.

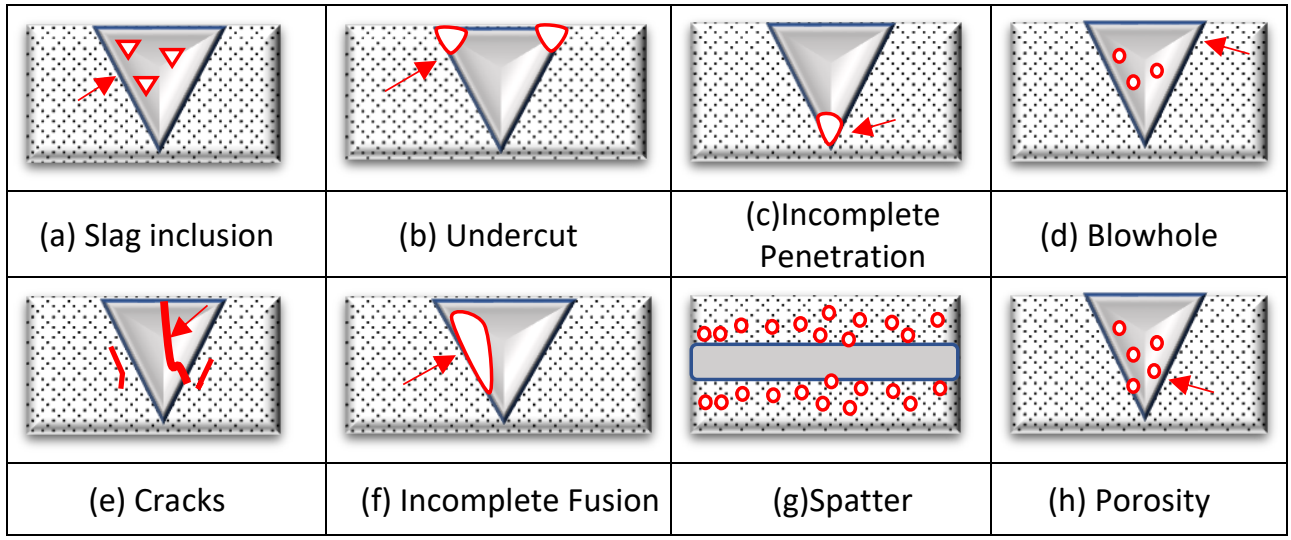

Figure 5. Eight Types of Defective Welding.

\subsection{YOLO-based Edge Object Detection}

Machine learning algorithms have been widely used for image recognition and can therefore be used for X-ray image defect recognition. To this end, this paper has developed a Yolo-based object detection model approach to detection, location and classification. It should provide a concise and precise description of the experimental results, their interpretation, as well as the experimental conclusions that can be drawn. Many different deep learning convolutional neural network (CNN) models, including DetectNet [15], R-CNN [21], GoogleNet [21, 22] and YOLO [2] have been proposed for object detection segmentation. In this section, the Region-based CNN (R-CNN) is used to determine the bounding box of object detections in RT images. The modified YOLO architecture evolved from the YOLOv3 model and replace the Full Convolutional Neural Network (FCN) framework for defective object detection in aviation maintenance RT images. The experiment has developed an efficient and powerful modified YOLOv4 algorithm object detection model. Machine inspection of RT images entails detecting the presence of defects, providing the type of part defect and the location or area of the defect, and issuing the type of defect. RCNN-based edge object detection can be used to train a super-fast and accurate object detector using NVIDIA GeForce GPUs [23]. The neural network layer learns attention mechanism object detection using a region proposal network (RPN). The innovative deep learning aerospace NDT models are therefore flexible and robust automatic object detection models that can be applied to R-CNN layer bypass hidden neural networks [24]. In addition to the modified R-CNN models, an ordinary modified YOLO object detector is 
composed of five parts; the input RT images, Backbone, Neck and Dense Prediction, which are shown in Figure 6.

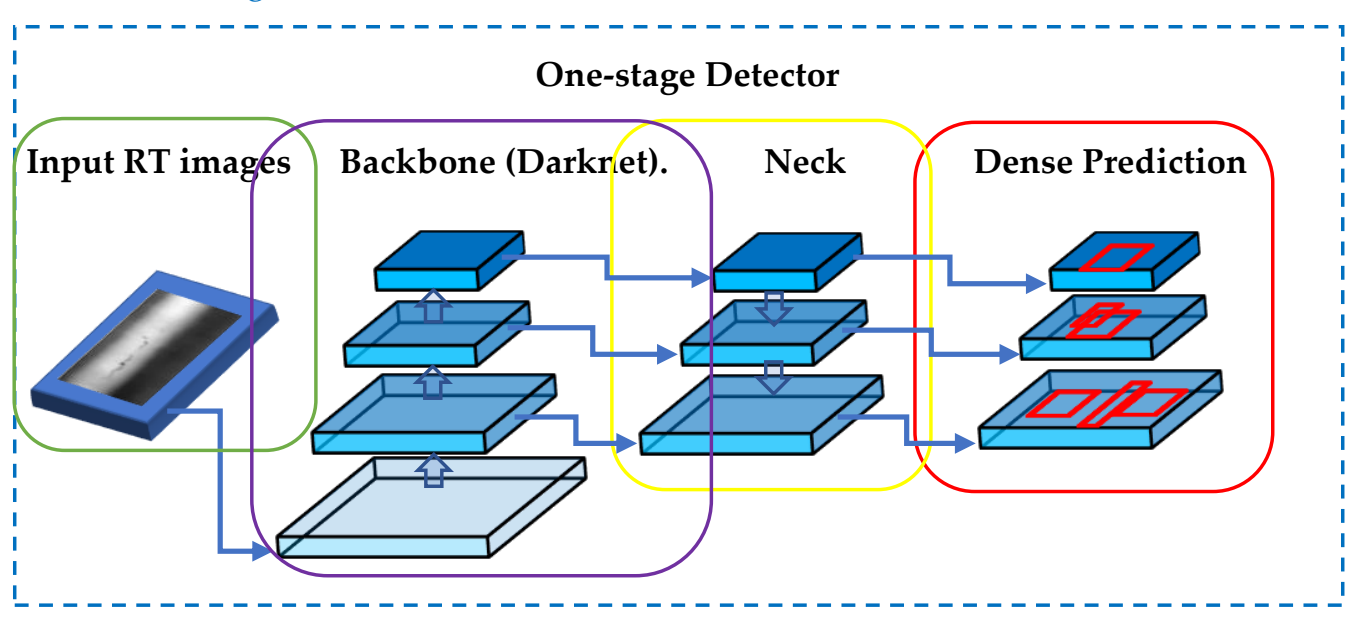

Figure 6. One-stage Detector parts.

The differentiates types of defect rating experimental parameters is used to provide the bounding-box object detection for the design of convolutional neural networks [9] for each layer. The normal CNN is extended to allow marking of the defect feature maps using on pooling layers, which could lead to faster convolution speed and better accuracy [10]. In YOLOv4 model, there is only few convolution layers, and the size of the output feature map is controlled by the backbone, neck and dense prediction one-stage neural network parts. This deep learning modified model is further developed to enhance the recognition of multiple objects.

\subsection{Optimal Object Detection for YOLOv4 algorithm}

In deep learning models, some research has focused on finding the best activation function. A good activation function allows the gradient to propagate more efficiently without incurring too much extra computational cost. Generally speaking, conventional object detectors are trained offline by the CPU. Therefore, this advantage is used to develop better training models for deep learning, allowing object detectors to achieve better accuracy without increasing the cost of inference. This paper refers to these methods that only change the training strategy or only increase the training cost as "bag of freebies" [25]. As a result, RT images unbiased classification for 8 types of object detection feature maps can be obtained. The YOLOv4 as depicted employs layers to realize an LRN (local area response normalization) [26] and uses a local neuron net to establish a set of active competition mechanisms in an attempt to suppress negligible small feedback neurons for the improvement of learning performance. The SPP module proposed by the He et al. [16, 27] changes the one-dimensional feature vector proposed by the SPP module through the output, which is not feasible for application in a fully convolutional network (FCN). Thus, in the design of YOLOv4, Redmon and Farhadi improve SPP module to the concatenation of max-pooling outputs with kernel size $\mathrm{k} x \mathrm{k}$, where $\mathrm{k}=(1,5,9,13)$ interval value, and stride equals to 1 [2]. Under this design, a relatively large $\mathrm{k} x \mathrm{k}$ max-pooling effectively increase the receptive field of backbone feature.

In the innovation one-stage-net model, there is only one convolution layer, and the size of the output feature map is controlled by adjusting the convolution step. So, there is no particular restriction on the size of the input image. In the flowchart, the RT input image is shown in $640 \times 480$ pixels as an example. Under the YOLOv4 algorithm small size feature map is used to detect large size objects, while the large size feature map detects small size objects. The output dimension of the feature map is $\mathrm{N}^{*} \mathrm{~N}^{*}\left[3^{*}(4+1+80)\right]$, there are 3 anchor boxes with 4-dimensional predicted values for each anchor box. If using for the GPU compute use a small number of groups (1-8) in convolutional layer CSPResNeXt50. 
The CSPResNext50 contains only 16 convolutional layers 3 x 3, a 425 x 425 receptive field and 20.6 M parameters, while CSPDarknet53 contains 29 convolutional layers $3 \times 3$, a 725 x 725 receptive field and $27.6 \mathrm{M}$ parameters [2].

\section{Experimental Result}

The CNN-based deep learning object detection and inspection system is designed to augment the capability of a human inspector to speed up the inspection task and to reduce the likelihood of errors. The modified YOLOv4 after training and validation is thus implemented in an embedded processor to perform the defect detection and image segmentation of X-ray images for NDT. The embedded platform that is adopted is the NVIDIA ${ }^{\circledR}$ Jetson $^{\mathrm{TM}}$ TX2 platform hardware which is responsible to perform the on-line inference for defect identification. The inference system is used to upgrade the factory NDT machine equipment, that helps the engineer to perform inspection all $\mathrm{X}$-ray images more efficiently.

\subsection{System Setup}

The main operation software is Ubuntu 16.04 version, together with additional installations of the NumPy software for $\mathrm{C}++$ and Python programming language interface and opensource software based on the YOLOv4 algorithm under the deep learning system to provide multi-class training and testing with the deep learning GPU training system. The processing chain for deep learning is described, as shown in Section 3. In the iterations of training, the optimal onetime iteration convergence curve in the trained model is selected as the data for the convergence model. ported to the NVIDIA ${ }^{\circledR}$ Jetson $^{\mathrm{TM}} \mathrm{TX} 2$ to perform inference task through YOLOv4 neural network. This innovative YOLOv4 deep learning object detection, classification and recognition module for LMDB data stored in a .txt file data format and labeled defect feature maps are trained to identify the object localization bounding box. This allows the detection of objects outside the normal environment. In addition, batch normalization calculates activation statistics for 8 different images on each layer, and successfully marks object feature map segmentation. This significantly reduces the need for large and small batches. The host computer is equipped with an 8-core Intel i7-7700K CPU and an NVIDIA-GTX 1660Ti GPU core graphics card. The host is responsible for the training of the neural network. The host computer is connected to an external extension, the NVIDIA ${ }^{\circledR}$ Jetson $^{\mathrm{TM}}$ TX2 embedded development board, to perform the inference tasks. The host computer is responsible for the entire data processing and training task. Once the model has been trained and validated in the host, it is compiled and ported to the NVIDIA ${ }^{\circledR}$ Jetson $^{\mathrm{TM}} \mathrm{TX} 2$ embedded development board for inference.

\subsection{Training Optimization}

The basic goals are fast running speed of neural networks, optimization of production systems and parallel computing, rather than theoretical metrics of low computational effort (BFLOP). This paper presents a selection of deep learning neural network models where the YOLOv4 neural network model is used. some snapshots of the X-ray image dataset are depicted, and section 3 of the paper illustrates the interface for the description of the image model. In the presence of defects, a category labeling bounding box is made and the regions within the region proposals box is used in the COCO and YOLO [28] algorithm architecture to perform feature value extraction operations on the trained. Then, the optimization loss algorithm is used to refine the model. The process can efficiently distinguish whether it is a defect or a background pixel [15] to reduce the error rate and correct the position of the bounding box by a linear regression model. In addition, the labeling tool named LabelImg software is used for object labeling and compilation as shown in Figure 7. The LabelImg software will automatically check whether there is a 
corresponding .xml file extension tag file in the system so that the overall training can be conducted more efficiently. Both training and testing are conducted in the determination of the neural network model. To this end, the image file data sets are divided into two folders among which $70 \%$ is used for training and $30 \%$ is used for testing.

More recently, research papers have continued to improve the IoU loss. For example, the GIoU loss [29] is to include the shape and orientation of the object in addition to the coverage area. As for the DIoU loss [30], it also considers the distance from the center of the object, while the CIoU loss [30], considers both the overlapping area, the distance between centroids and the aspect ratio. The experimental model in this thesis using CIoU can achieve better convergence speed and accuracy on the BBox regression problem. The experimental uses deep networks as a model to identify the RT image defect object localization window and bounding box. Attention modules often used in object detection are mainly divided into channel-based attention and point-based attention, which are represented by the Squeeze and Excite (SE) [31] and Spatial Attention Module (SAM) [4] attention models. In order to make the designed detector more suitable for training on a single GPU, the following additional design and improvements have been made to this model. The model introduces new data enhancement methods structure of the YOLOv4 model used in the experimental process is improved and optimized consists of Backbone: CSPDarknet53 [18], CutMix. Neck: SPP [32], PAN [3]. Detector: CutMix [33], Cross MiniBatch Normalization (CmBN), DropBlock regularization [34], and CIoU loss [30]. Head: YOLOv3 [2]. The loss function for target detection tasks usually consists of two components, Classification Loss Function and Bounding Box Regression Loss Function. The YOLOv4 use Bounding Box Regression Loss has evolved in the DIoU Loss (2020) and CIoU Loss (2020). Also, CmBN is defined as Cross Mini-Batch Normalization. This only collects statistics between mini batches within a single batch. These require careful adjustment of the loss function in order to converge the influence factor function more quickly on Equation (1) and (2) and enhance the ability to extract features.

$$
\begin{aligned}
\text { CIOU Loss } & =1-\operatorname{CIOU}\left(\text { IOU }-\frac{\text { Distance } 2^{2}}{\text { Distance } C^{2}}-\frac{v^{2}}{(1-\text { IOU })+v}\right) \\
\mathrm{v} & =\frac{4}{\pi^{2}}\left(\arctan \frac{w^{g t}}{h^{g t}}-\arctan \frac{w^{p}}{h^{p}}\right)^{2}
\end{aligned}
$$

Distance $\mathrm{C}=$ Minimum diagonal distance from external rectangle.

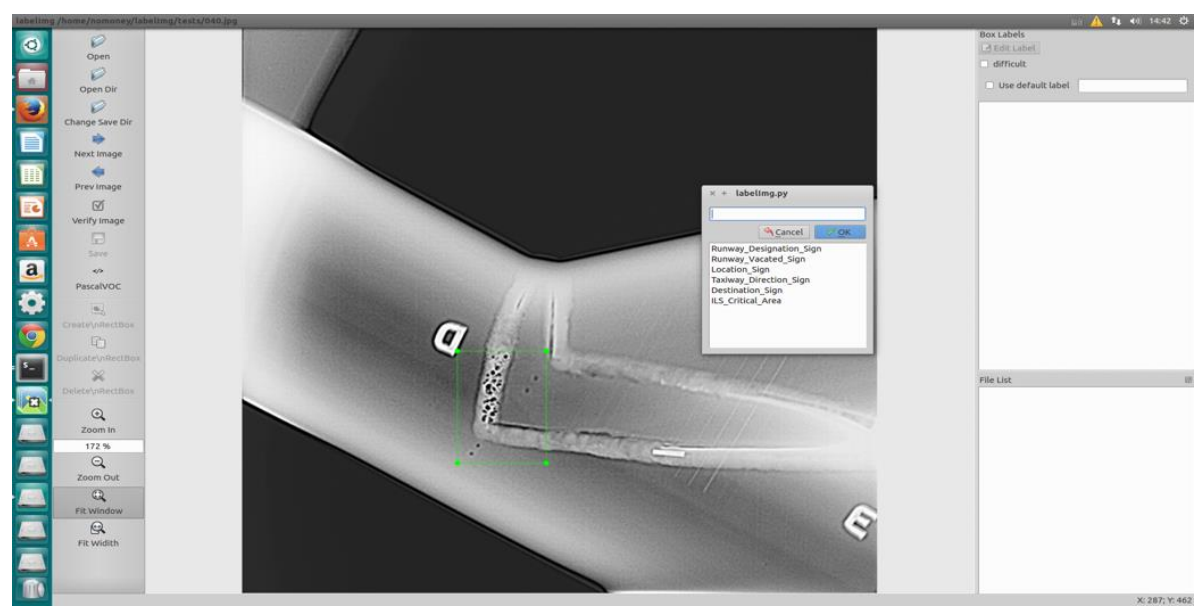

Figure 7. LabelImg software for object labeling and compilation. 


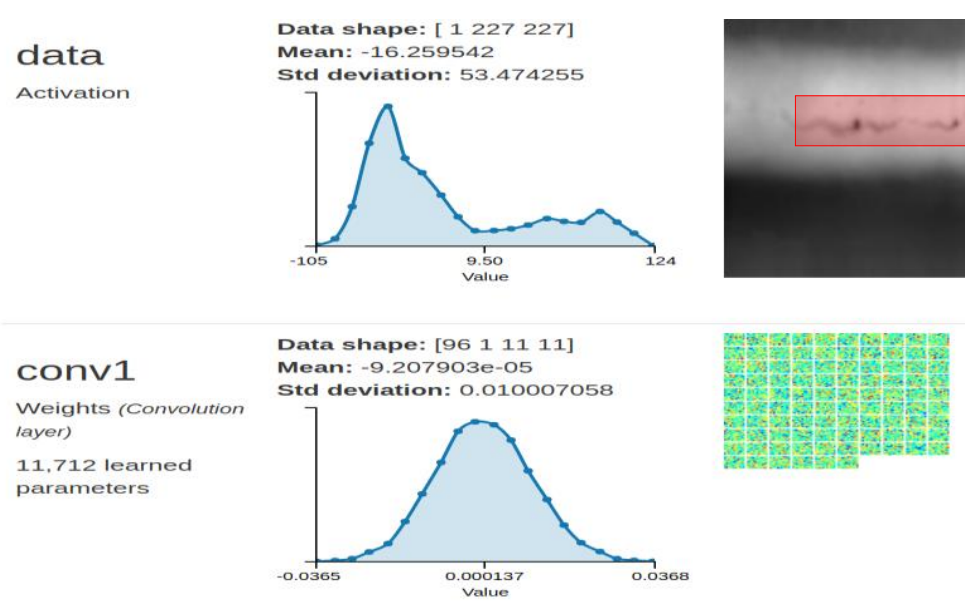

Figure 8. LabelImg software for object labeling and compilation.

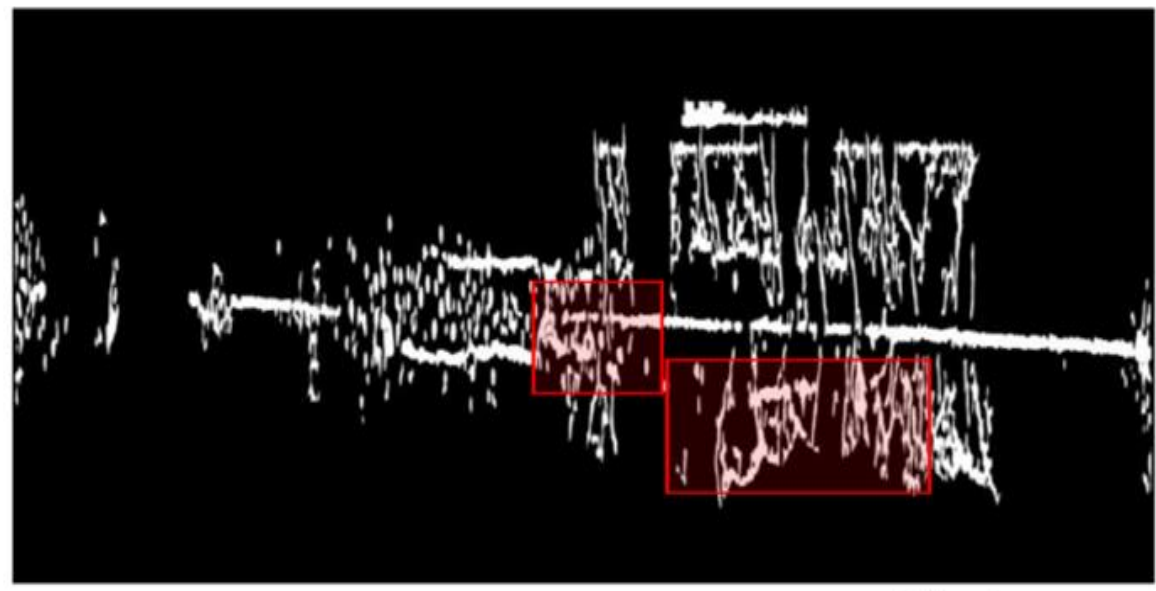

Figure 9. LabelImg software for object labeling and compilation.

\subsection{Self-Advocacy Training}

To overcome this attention-based neural network issue, this thesis employed a deep learning paradigm of optimal object detection tackling both single and multiple detection. The simple experiment compared with the different Fast CNN and YOLO structure models. Although the mAP (Mean-Average-Precision) results are listed in Table 1, the accuracy rate of YOLO was slightly higher than that of Fast RCNN, and the Loss score of Fast RCNN was higher than that of YOLO. However, the average elapsed times of the onestage methods were much less than the two-stage methods. Our YOLOv4 are located superior to the fastest detectors in terms of both speed and accuracy. The self-advocacy training (SAT) also represents a new data enhancement technique that operates in two forward-to-backward phases. In the first stage, the neural network alters the original image rather than the network weights. In this way, the neural network performs an adversarial attack on itself, altering the original image to create the deception that there is no desired object on the image. In the second stage, the neural network is trained to detect objects on that modified image in a normal way.

The operation follows the description in Figure 8, and typically the model is trained for more than 600 iterations as show in Figure 9. The self-advocacy trained neural network structure acts as a filter for the data. When convolving through the first layer, it is forced through a custom layer. The self-advocacy trained layer sharpens the image features so that the features become prominent. As a result, the error rate of model recognition is reduced by $1-2 \%$. 
Table 1. Inspection results of four methods.

\begin{tabular}{cccc}
\hline Method & $\begin{array}{c}\text { Accuracy } \\
(\mathbf{m A P})\end{array}$ & $\begin{array}{c}\text { Loss } \\
\mathbf{( \% )}\end{array}$ & $\mathbf{T}(\mathbf{S})$ \\
\hline Faster-RCNN & 0.78229 & 0.00238 & 0.2708 \\
Original Mask-RCNN & 0.79129 & 0.00238 & 0.3982 \\
YOLOv2 & 0.79229 & 0.0238 & 0.0298 \\
YOLOv3 & 0.72239 & 0.0238 & 0.0326 \\
YOLOv4 & 0.72416 & 0.0238 & 0.0235 \\
\hline
\end{tabular}

\section{Discussion}

The results of the thesis have made considerable efforts in order to improve the accuracy and efficiency of non-destructive testing of civil aircraft and engines at the D-Level inspection stage through the use of artificial intelligence techniques to benefit the method in the global aerospace industry. The aim of this paper is to provide a new model for deep learning to detect good results in aero-engine radiographic inspection systems to assist in the detection of defect damage in X-ray image files of RT. This is in part a result of the use of the YOLOv4 model based on powerful deep learning techniques to prevent human error in future non-destructive testing. To provide clarity on the main issues regarding the testing of various deep learning models, this paper provides relative experimental results for four well-known detection methods, demonstrating that YOLOv4 is slightly more accurate than all methods and scores slightly better than the various of RCNN models.

\section{Conclusions}

Considerable efforts have been made to improve the accuracy and efficiency of nondestructive inspection of jet engine fans through the use of artificial intelligence techniques in order to benefit the method in the aerospace industry. The aim of this paper is to provide a new method for testing the effectiveness of aero-engine radiographic inspection systems to detect defective damage from X-ray image files. This is in part a result of the use of the YOLOv4 model based on powerful artificial intelligence techniques to prevent future human error in non-destructive testing. In addition, the main types of YOLOv4 testing and training validation methods are analyzed and trialed in the paper. In addition, a case study of eight types of material defect classifier applications is presented to demonstrate the feasibility and effectiveness of the proposed quality validation method. The results achieved an average accuracy (mAP) of 0.9 on eight types of material defect classifier problems and required approximately $100 \mathrm{miles} / \mathrm{sec}$ to develop a deep learning-based inspection program to enhance non-destructive inspection work in civil aircraft repair shops. Fortunately, by replacing the inherent method with a deep learning model based on artificial intelligence, some benefits can be achieved in terms of time efficiency in non-destructive inspection and savings in labor resource consumption. The experimental results utilize deep learning techniques to determine bespoke model parameters to improve the quality assurance of NDT defects. The resulting weights were successfully deployed in $\mathrm{C}++$ code on a TX2 development board. The model was tested and validated to assist inspectors in performing diagnostic tasks on X-ray images in civil aircraft repair shops. In particular, the results show that a deep learning neural network-based inspection system can enhance the overall inspection system, reducing human workload and ensuring quality.

Author Contributions: Author Contributions: Zhi-Hao Chen, performed the modified YOLOv4 design, have written $\mathrm{C}++$ code and installed in the Jetson ${ }^{\mathrm{TM}} \mathrm{TX} 2$ embedded computer and wrote the paper. Jyh-Ching Juang supervised the project. All authors have read and agreed to the published version of the manuscript. 
Funding: This research received no external funding.

Conflicts of Interest: The authors declare no conflict of interest. The funders had no role in the design of the study; in the collection, analyses, or interpretation of data; in the writing of the manuscript, or in the decision to publish the results.

\section{Reference}

1. From Wikipedia, t. f. e. Southwest Airlines Flight 1380, April 17, 2018.

2. Redmon, J.; Farhadi, A., Yolov3: An incremental improvement. arXiv preprint arXiv: 1804.027672018.

3. Liu, S.; Qi, L.; Qin, H.; Shi, J.; Jia, J. In Path aggregation network for instance segmentation, Proceedings of the IEEE conference on computer vision and pattern recognition, 2018; 2018; pp 8759-8768.

4. Woo, S.; Park, J.; Lee, J.-Y.; Kweon, I. S. In Cbam: Convolutional block attention module, Proceedings of the European conference on computer vision (ECCV), 2018; 2018; pp 3-19.

5. Yao, Z.; Cao, Y.; Zheng, S.; Huang, G.; Lin, S., Cross-iteration batch normalization. arXiv preprint arXiv:2002.05712 2020.

6. Shin, H. C.; Roth, H. R.; Gao, M.; Lu, L.; Xu, Z.; Nogues, l.; Yao, J.; Mollura, D.; Summers, R. M., Deep Convolutional Neural Networks for Computer-Aided Detection: CNN Architectures, Dataset Characteristics and Transfer Learning. IEEE Trans Med Imaging 2016, 35, (5), 1285-98.

7. Pontecorvo, J. A. MSG-3-A Method For Maintenance Program Planning; 0148-7191; SAE Technical Paper: 1984.

8. Mathaisel, D. F., A lean architecture for transforming the aerospace maintenance, repair and overhaul (MRO) enterprise. International Journal of Productivity and Performance Management 2005.

9. Sikora, R.; Baniukiewicz, P.; Chady, T.; Łopato, P.; Piekarczyk, B.; Psuj, G.; Grzywacz, B.; Misztal, L. In Detection and classification of weld defects in industrial radiography with use of advanced Al methods, 2013 Far East Forum on Nondestructive Evaluation/Testing: New Technology and Application, 17-20 June 2013, 2013; 2013; pp 12-17.

10. Board, N. T. S., DCA18MA142 SWA1380 INVESTIGATIVE UPDATE. In 2018.

11. Komar, M.; Yakobchuk, P.; Golovko, V.; Dorosh, V.; Sachenko, A. In Deep Neural Network for Image Recognition Based on the Caffe Framework, 2018 IEEE Second International Conference on Data Stream Mining \& Processing (DSMP), 21-25 Aug. 2018, 2018; 2018; pp 102-106.

12. Gong, Y.; Shao, H.; Luo, J.; Li, Z., A deep transfer learning model for inclusion defect detection of aeronautics composite materials. Composite Structures 2020, 252, 112681.

13. Liu, W.; Anguelov, D.; Erhan, D.; Szegedy, C.; Reed, S.; Fu, C.-Y.; Berg, A. C. In SSD: Single Shot MultiBox Detector, Computer Vision - ECCV 2016, Cham, 2016//, 2016; Leibe, B.; Matas, J.; Sebe, N.; Welling, M., Eds. Springer International Publishing: Cham, 2016; pp 21-37.

14. Kannadaguli, P. In YOLO v4 Based Human Detection System Using Aerial Thermal Imaging for UAV Based Surveillance Applications, 2020 International Conference on Decision Aid Sciences and Application (DASA), 2020; IEEE: 2020; pp 1213-1219.

15. Yang, F.; Fan, H.; Chu, P.; Blasch, E.; Ling, H. In Clustered Object Detection in Aerial Images, 2019 IEEE/CVF International Conference on Computer Vision (ICCV), 27 Oct.-2, 2019; 2019; pp 8310-8319.

16. Ren, S. Q.; He, K. M.; Girshick, R.; Sun, J., Faster R-CNN: Towards Real-Time Object Detection with Region Proposal Networks. leee Transactions on Pattern Analysis and Machine Intelligence 2017, 39, (6), 1137-1149.

17. Redmon, J.; Farhadi, A. In YOLO9000: better, faster, stronger, Proceedings of the IEEE conference on computer vision and pattern recognition, 2017; 2017; pp 7263-7271.

18. Wang, C.-Y.; Liao, H.-Y. M.; Wu, Y.-H.; Chen, P.-Y.; Hsieh, J.-W.; Yeh, I.-H. In CSPNet: A new backbone that can enhance learning capability of CNN, Proceedings of the IEEE/CVF conference on computer vision and pattern recognition workshops, 2020; 2020; pp 390-391.

19. Xiao, Z. F.; Gong, Y. P.; Long, Y.; Li, D. R.; Wang, X. Y.; Liu, H., Airport Detection Based on a Multiscale Fusion Feature for Optical Remote Sensing Images. leee Geoscience and Remote Sensing Letters 2017, 14, (9), 1469-1473. 
20. Henry, G., Howard Chu on Lightning Memory-Mapped Database. leee Software 2019, 36, (6), 83-87.

21. Zhang, J.; Cosma, G.; Watkins, J., Image enhanced mask R-CNN: A deep learning pipeline with new evaluation measures for wind turbine blade defect detection and classification. Journal of Imaging 2021, 7, (3), 46.

22. Aswathy, P.; Siddhartha; Mishra, D. In Deep GoogLeNet Features for Visual Object Tracking, 2018 IEEE 13 th International Conference on Industrial and Information Systems (ICIIS), 1-2 Dec., 2018; 2018; pp 60-66.

23. Buck, I., GPU Computing: Programming a Massively Parallel Processor. In International Symposium on Code Generation and Optimization (CGO'07), 2007; pp 17-17.

24. Subramanian, A.; Schwartz, R. In Reference-free inference of tumor phylogenies from single-cell sequencing data, 2014 IEEE 4th International Conference on Computational Advances in Bio and Medical Sciences (ICCABS), 2-4 June 2014, $2014 ; 2014 ;$ pp 1-1.

25. Chang, Y.; Huang, J. C.; Su, L.; Chen, Y. A.; Chen, C.; Chou, C. In Localized surface plasmon coupled fluorescence fiber-optic biosensor for severe acute respiratory syndrome coronavirus nucleocapsid protein detection, 2009 14th OptoElectronics and Communications Conference, 13-17 July 2009, 2009; 2009; pp 1-2.

26. Saari, I. S.; Mahmud, Z.; Abdullah, N. N. In Diagnosis of response behavioural patterns towards the risk of Pandemic Flu Influenza A (H1N1) of urban community based on Rasch measurement model, 2012 International Conference on Statistics in Science, Business and Engineering (ICSSBE), 10-12 Sept. 2012, 2012; 2012; pp 1-6.

27. Bochkovskiy, A.; Wang, C.-Y.; Liao, H.-Y. M., Yolov4: Optimal speed and accuracy of object detection. arXiv preprint arXiv:2004.10934 2020.

28. Lin, T.-Y.; Maire, M.; Belongie, S.; Hays, J.; Perona, P.; Ramanan, D.; Dollár, P.; Zitnick, C. L. In Microsoft coco: Common objects in context, European conference on computer vision, 2014; Springer: 2014; pp 740-755.

29. Rezatofighi, H.; Tsoi, N.; Gwak, J.; Sadeghian, A.; Reid, I.; Savarese, S. In Generalized intersection over union: $A$ metric and a loss for bounding box regression, Proceedings of the IEEE/CVF Conference on Computer Vision and Pattern Recognition, 2019; 2019; pp 658-666.

30. Zheng, Z.; Wang, P.; Liu, W.; Li, J.; Ye, R.; Ren, D. In Distance-loU loss: Faster and better learning for bounding box regression, Proceedings of the AAAI Conference on Artificial Intelligence, 2020; 2020; pp 12993-13000.

31. Hu, J.; Shen, L.; Sun, G. In Squeeze-and-Excitation Networks, 2018 IEEE/CVF Conference on Computer Vision and Pattern Recognition, 18-23 June 2018, 2018; 2018; pp 7132-7141.

32. He, K.; Zhang, X.; Ren, S.; Sun, J., Spatial pyramid pooling in deep convolutional networks for visual recognition. IEEE transactions on pattern analysis and machine intelligence 2015, 37, (9), 1904-1916.

33. Yun, S.; Han, D.; Oh, S. J.; Chun, S.; Choe, J.; Yoo, Y. In Cutmix: Regularization strategy to train strong classifiers with localizable features, Proceedings of the IEEE/CVF International Conference on Computer Vision, 2019; 2019; pp 6023-6032.

34. Ma, N.; Zhang, X.; Zheng, H.-T.; Sun, J. In Shufflenet v2: Practical guidelines for efficient cnn architecture design, Proceedings of the European conference on computer vision (ECCV), 2018; 2018; pp 116-131. 